\title{
Sustaining health improvement activities delivered in English professional football clubs using evaluation: A short communication
}

Andy Pringle*, Daniel Parnell, Zoe Rutherford, Jim McKenna, Stephen Zwolinsky and Jackie Hargreaves.

Centre for Active Lifestyles, Research Institute of Sport, Physical Activity and Leisure, Leeds Beckett University, LEEDS, UK. LS6 3QS.

\begin{abstract}
It has been suggested that football and communities are inextricably linked. Healthy lifestyles are an important component in maintaining the sustainability of local communities, not least, because a convincing evidence base supports the holistic benefits that can be derived from health enhancing behaviours such as, regular physical activity. As such, efforts to promote health improvement through sport and physical activity include those interventions delivered in professional sporting settings. Johnman and colleagues ${ }^{1}$ have heralded sports clubs as important venues for the delivery of health improvement interventions for a range of groups across local communities. This includes health improvement activities delivered in professional football club community schemes. While exemplary practice shows how health improvement programmes can be implemented and evaluated, our experience and engagement with professional football club community schemes supports the notion that more needs to be undertaken to help clubs develop monitoring and evaluation strategies in order to assess the impact of their health improvement programmes. In our short communication, we share our plans for helping two professional football clubs develop their monitoring and evaluation strategies for their community health promotion programmes. Potential outcomes emerging from this process are two-fold. (I) To help club-community schemes in-build and sustain monitoring and evaluation practices into their future healthimprovement provision. (II) To use the impact and process outcomes emerging from programme evaluations, to successfully secure the necessary resources to sustain future health improvement activities for their local communities. Outcomes emerging from this study will be of interest to football clubs and evaluators alike, as they seek to develop evaluation strategies for their health improvement programmes.
\end{abstract}

Key words football, health, public health, intervention, evaluation, sustainability 
* Corresponding author: a.pringle@leedbeckett.ac.uk

\section{Introduction}

Johnman and colleagues have heralded sports clubs as important venues for the delivery of health improvement interventions for a range of groups across local communities. ${ }^{2}$ This includes health improvement activities delivered in professional football club community schemes. While good practice shows how health improvement programmes can be evaluated $^{3}$, our experience and engagement with professional football clubs supports the notion that more needs to be done to help clubs develop monitoring and evaluation strategies in order to assess the impact of their health improvement programmes. In our short communication, we share our plans for helping two professional football clubs to develop their monitoring and evaluation strategies to sustain their health promotion programmes for their local communities.

\section{Background}

Healthy lifestyles are an important component in maintaining the sustainability of local communities, not least because it allows individuals to both maintain and improve their health and wellbeing holistically. ${ }^{4}$ Chronic health conditions such as cancer, cardiovascular disease, diabetes and chronic respiratory disease are now grouped together in public health terms as 'non-communicable diseases' (NCDs). ${ }^{5}$ NCDs are thought to be responsible for 36 million deaths worldwide each year and evidence shows that around 9 million of these occur before the age of $60 .^{6}$ Modifiable lifestyle risk factors underpinning NCDs include physical (in)activity, poor diet, smoking and the harmful use of alcohol. ${ }^{7}$ These behaviours can bring about metabolic/physiological changes that increase the risk of developing NCDs including, elevated blood pressure, overweight/obesity, hyperglycaemia and hyperlipidaemia. ${ }^{8}$ However, even relatively small changes in these modifiable lifestyle risk factors can produce substantial health benefits, ${ }^{9}$ nevertheless, eliciting and sustaining long term positive change in these behaviours remains problematic from a public health perspective. At the heart of many of these conditions, is physical (in)activity, where a convincing evidence base supports the role of a physically active lifestyle in the prevention and management of a range of chronic conditions. ${ }^{10}$ It is then no surprise that the delivery of healthy and physically active lifestyles through health improvement activities is an important part of health policy. ${ }^{11}$ Despite such efforts, the 'health of the nation' remains a cause for concern; ${ }^{12}$ as does the need to 
effectively intervene with health improvement opportunities, including those involving the promotion of sport and physical activity. ${ }^{13}$

With those thoughts in mind, a leading Public Health body, the Royal Society for Public Health have recently suggested that the "settings in which we live, offer huge opportunities and threats to our health. Addressing the social determinants of health is vital to improving population health and reducing health inequalities, and utilising a settings based approach to health provides a way to promote, improve and protect health and tackle health inequalities." 14 Further, interventions that create and strengthen a diverse natural social network, increase the availability of social support and reduce negative interactions are thought to be a "best buy" for public health. ${ }^{15}$ Therefore, as football and community settings are thought to be inextricably linked, ${ }^{16}$-and with health and lifestyle intervention of uppermost importance, ${ }^{17}$ recreational and professional football settings offer one way for connecting individuals and communities with health improvement opportunities. ${ }^{18}$ This not only includes priority groups from across the life course (children/young people, adults and older adults), ${ }^{19}$ but also, those groups who have been labelled 'hard to engage' or 'unreached' (including, men, substance users and individuals who do not access conventional health services). ${ }^{20}$

Indeed, the appeal of sporting clubs has been heralded as having important potential global appeal in public health terms. ${ }^{21}$ As a result, locating health improvement programmes within professional football clubs offers both participants and providers with an attractive proposition and a setting for reaching communities with health behavioural change programmes. $^{22}$ The reasons appear to be multifaceted and include, the reach of the activity itself (i.e. the football//sport), the affiliation of individuals to their football club, the venue of delivery and processes by which health programmes are packaged and delivered. ${ }^{23}$ Moreover, it should be noted that the appeal of football clubs as settings for health improvement extends to fans, non-fans and in some cases, those who are not interested in football, ${ }^{24}$ thus endorsing the potential reach suggested by Johnman, Mackie and Sim. ${ }^{25}$

The importance of selecting the most appropriate settings for reaching individuals with health improvement opportunities takes on an added importance when the current fiscal climate is considered. Reductions in funding for health services ${ }^{26}$ and local authority led sport ${ }^{27}$ mean there is not only increased competition on those football clubs attempting to secure funding, 
but arguably a heightened need to demonstrate how such resources are being used effectively and efficiently. In demonstrating such an impact, the requirement to evaluate has never been more important. ${ }^{28}$ This is not only about demonstrating the impact and the process by which programmes have their effect, ${ }^{29}$ but also securing the necessary resources to help sustain effective programmes once the initial 'start up' funding has become extinct. ${ }^{30}$

Notwithstanding existing good practice in both research and evaluation, ${ }^{31}$ our experience of reviewing funding applications for football-led health improvement programmes and dialogue/interaction with clubs, led us to conclude, that more needs to be undertaken to help some football clubs develop effective and workable evaluation strategies. In doing so, ceasing the potential to demonstrate the impact of their programmes and the ability to secure resources to sustain their programmes, and this provides the 'raison detre' for our study. We feel that this paper will be of interest to our colleagues in professional football club community schemes that face the prospect of developing and implementing evaluation strategies either independently or in partnership evaluation arrangements with their 'local evaluators'. ${ }^{32}$ Moreover, our paper compliments the available guidance that helps to shape evaluation strategies and which provides important practical advice and considerations for those working in this important area. ${ }^{33}$

\section{Funding context}

In providing the context to our work, we provide the following information and background. Subsequent to an internal funding call to develop sustainable practice in local communities from the Higher Education Innovation Fund (HEIF) and with the support of the Leeds Beckett University Enterprise Team, our researchers were successful in securing funding of around $£ 10,000$. Our funding proposal outlined how we would use the resources secured to: (I) help professional football clubs develop effective (valid, reliable and practical evaluation strategies) for their health improvement programmes and (II) help sustain the future delivery of health improvement programmes offered by professional football clubs to their local communities. While we received funding to support the work described here, there are examples where we have performed similar evaluations using institutional resources and for the purposes of research and investigation. ${ }^{34}$ As such, this raises the possibility that clubs might work with their local university department or other local evaluators in a similar fashion. 


\section{Intervention context}

As part of our existing partnerships, we are in this example, working with two professional clubs located in the English East Midlands, where we have been developing evaluation strategies for their health improvement programmes. We use the following sections to introduce the clubs, examples of their health improvement programmes and examples of their evaluation needs. Our first club is Burton Albion FC, where our collaboration with their Community Trust extends several years through co-author Parnell. Examples of earlier outcomes emerging from this partnership have been published elsewhere. ${ }^{35}$ Our second partner is Notts County in the Community (the community arm of Notts County Football $\mathrm{Club}$ ), where our previous alliance extends through co-author Rutherford. Examples of outcomes emerging from this alliance have similarly been published elsewhere in the literature. ${ }^{36}$ Both of these organisations are registered charities and are attached to their respective professional football clubs. In both cases, programmes are packaged and delivered using all the branding of the football clubs and we offer some examples of the interventions being delivered and how they aspired to meet the needs of participants in local and neighbouring communities.

Understanding the importance of promoting physical activity across the lifespan, we provide three examples from Burton Albion. Our first example 'Head for Goal' aims to promote positive mental well-being for young adult men 16-24 years, through a weekly programme of football delivered at local community venues. Secondly, 'Golden Goal' is a programme of physical and social activity delivered for 'older-adults' 55 years and older. Thirdly, the club have an extensive physical education and school sport programme that delivers an extended school curriculum. Similarly at Notts County interventions are delivered across the lifespan. Firstly, recognizing the increase and impact of overweight and obesity as a health improvement issue, the Notts County's, 'Motivate' programme offers both adult men and women with a programme of weight management classes. Activities are delivered by club coaches and nutritionists in local community venues within the City of Nottingham. Secondly, continuing with the adult population, 'Energise' is an example of an on-site, 18week workplace health and wellbeing programme, delivered to employees during lunch breaks and evenings, using safe, effective and enjoyable physical activity. Activity sessions are delivered by FITC's experiences coaches. Thirdly, recognizing the importance of the health of young adult men, 'On The Ball', is a health improvement programme that has engaged over 250 young men over the last five years. The programme provides weekly 
football sessions for a core of 40 participants who continue to activity sessions at a local community facility. In both clubs, the need to evaluate impact and process outcomes was fundamental to demonstrating intervention effect and for sustaining health improvement provision in the future.

Our team have worked with both clubs to establish how sustainable and workable evaluation strategies can be developed and implemented for their health improvement programmes. In describing this process, we have used components of 'intervention mapping' as an organising framework. ${ }^{37}$ Intervention mapping is an iterative process that has been used for planning and implementing health improvement programmes and their evaluations. We have used elements of this framework to both organise and report the development and implementation processes (i.e. evaluation needs, planning and implementation) that we put in place when developing the monitoring and evaluation strategies for health improvement programmes.

\section{Evaluation Needs and Planning}

Through our collaborations, the two participating clubs expressed the need for support with the development of their monitoring and evaluation strategies. Following existing practices for developing evaluation strategies of health improvement programmes of this nature, ${ }^{38}$ our evaluation team met the management at the respective clubs to discuss their current projects and their monitoring and evaluation activities. This included the project outcomes, current monitoring and evaluation processes, barriers to performing evaluation activity, service delivery arrangements and the stakeholders involved in the interventions. Following this, a second meeting was set up with the practitioners specifically involved in the delivery of interventions described previously (i.e., the coaches). These discussions also considered future funding targets and shared partnership opportunities, alongside broader engagement with the club's stakeholders through their local steering groups. During these discussions, staff expressed their 'evaluation needs'. While these were not all prevalent in our two clubs, examples of the kind of 'needs' which emerged included capacity (i.e. time to procure and develop evaluation protocols, collect analyse and report data), commitment (i.e pressures on staff to deliver interventions leaving less time for evaluation activities) and capability (i.e. skills to develop evaluation methodologies and instrumentation, implementing data protection protocols, obtain research ethics clearance and develop protocols for collecting and analysing data and interpreting and writing up findings/results from evaluations). ${ }^{39}$ Using information from these discussions, the research team developed evaluation strategies, 
which were bespoke to the respective interventions and provided support to help address some of the needs expressed by staff. These were prepared with the purpose of measuring and evidencing the impact of the respective health improvement programmes.

Staff at the clubs, also highlighted the need to demonstrate how programmes' impacted on the health profiles of local priority groups. For adult programmes, we advised on the following impact outcomes which have been used in the evaluation of community based health improvement programmes, including those delivered in football-led settings. ${ }^{40}$ These measures included, the collection of the following data and helped to establish the reach of programmes: Demographic profiles, (including age, gender, ethnicity and post-code); affiliation to football/ or football clubs (i.e. football fan, non-fan, fan of host club, fan of other clubs); if the activity adopted was new or replacement activity (i.e. if the adopted physical activity programme was a substitute or a brand new activity) and lifestyle profiles (including physical activity, healthy eating, smoking, alcohol and body mass index). These were supplemented by general measures of health status used elsewhere in the literature ${ }^{41}$ along with use and uptake of primary health care services (i.e consulting GP/Primary Care Services), as appropriate. ${ }^{42}$

Our experience of collecting this information - pre-post-intervention - has been helpful in: (I) building up a profile of the reach of programmes when recruiting local populations, including 'priority groups' and their baseline health profiles and (II) confirming the extent to which such provisions have been effective in facilitating 'change' in health status/profiles. In our two examples, measures were adapted to suit the target audience; including young people, older people or those constituents who are known to experience difficulties engaging evaluations. ${ }^{43}$ Along with these adapted measures, instrumentation and protocols were developed that were more suited for securing data on impact outcomes among these groups. (i.e. when assessing changes in the PA levels of young people). Moreover, the basic service level evaluation described above can be supplemented with additional evaluation activities as required by particular clubs. For instance, where appropriate, the self-report measures outlined can be supplemented with objective measures to assess impact outcomes. Supported by trained researchers, we have been able to offer specific cardiovascular fitness tests; tests for functional capacity; accelerometry to assess the frequency, intensity and duration of physical activity being performed; measurements of overweight/obesity and cardiovascular 
risk (e.g. waist circumference, \% body fatness, blood pressure and cholesterol) and reported elsewhere. ${ }^{44}$

In our discussions, staff expressed the value of (I) obtaining insights into how interventions have an impact and (II), the participant's perspectives of engaging programmes. With those thoughts in mind, participant case studies are often required within funding applications and/or reports to funders, as well in the preparation of publicity for programmes. As such, and in order to investigate the process by which interventions achieve their impact outcomes $^{45}$, we raised the feasibility of performing interviews with key stakeholders involved in the interventions. In doing so, we aspire to secure the perspectives of the key participants involved in the interventions. This is being undertaken using procedures adopted for process evaluations in football-led health improvement programmes ${ }^{46}$ and national community physical activity programmes. ${ }^{47}$ However, by way of example, interviews or focus groups aspired to investigate participant's experiences of attending interventions and what key design factors were important in facilitating 'change' across the behavioural continuum. That is, what key design characteristics (i.e. the programme offered, the process of delivery, the promotions and publicity used, the place and people involved in the delivery and the price of the programme) were important/less important in helping people learn about, start, stick and retrial interventions as appropriate. ${ }^{48}$ Prior to any data collection, the research team secured university research ethics clearance for the evaluation activities to take place.

Following the development of the evaluation packages, the research team organised project specific training days in order to build capacity within existing practitioners at each club who would become 'leads for evaluation'. This training typically lasted one day and was delivered on site and in advance of the evaluations commencing. The training covered a range of topics including ethics, consent (i.e informing participants of their rights when engaging evaluations), data protection (i.e keeping evaluation data secure), administration of the instrumentation (i.e how to introduce the evaluation to participants and collect data) and handling of the data (i.e setting up spread sheets for inputting evaluation data) as performed in evaluations of football-based health improvement programmes. ${ }^{49}$ The research team provided on-going support through meetings, phone calls and/or video calls up during data collection and quality assurance checks of the data were performed at regular intervals. As is standard practice in guidance ${ }^{50}$ and prior to the commencement of data collection, 
instrumentation was piloted and the application of instrumentation practiced in line with public health guidance on evaluation. ${ }^{51}$

\section{Evaluation Implementation}

On commencement of the interventions, a member of the research team attended and supported baseline data collection in a collaborative and supportive way to ensure correct techniques were being performed. In line with guidance issued elsewhere ${ }^{52}$ regular communications took place between the research team and the 'lead evaluator' (at the club) and this continued throughout the lifespan of the health improvement project, as recommended elsewhere. ${ }^{53}$ Furthermore, in line with evaluation needs expressed by the staff, the research team would later provide analysis and reports on the data collected. Using a framework from the literature ${ }^{54}$, our participating clubs have the opportunity to utilize the impacts of their respective evaluation activities in a number of ways. Firstly from a formative perspective, clubs can identify and take remedial action on parts of the programme which are not working as well and actions can be taken to refine provisions. Similarly, evaluations provide an opportunity to confirm which parts of the intervention are working effectively and the reasons for this. Secondly, where appropriate, clubs have the opportunity to develop knowledge and the necessary skills and expertise to in-build/procure monitoring and evaluation activities as part of their future practice. Thirdly, from an ethical perspective, to establish if interventions are effective and that individuals are experiencing the anticipated benefits of attending health improvement programmes. Finally, sustainability, to use the impacts emerging from these evaluations to make the case to local commissioners/national funding agencies to sustain future provisions. Given the competition for scarce public health resources, the outcomes emerging from well thought out evaluation designs have the potential to make a convincing case for future funding, a key evaluation need expressed by staff in the clubs.

\section{Conclusion:}

We set out to outline the importance of helping professional football club/community schemes sustain their health improvement interventions through effective, acceptable and adaptable evaluation strategies. As the programme of work develops, we aim to disseminate detailed findings of the effect of the respective programmes, how these health improvement provisions were implemented, along with reports on how evaluation outcomes were used to sustain existing/develop new health improvement programmes. 


\section{Acknowledgements}

In preparing this manuscript the authors most gratefully acknowledge the staff of Burton Albion FC and Notts County FC, as well as Leeds Beckett University and the Higher Education Innovation Fund who provided the resources for this work to be undertaken.

\section{Notes}

\footnotetext{
${ }^{1}$ Johnman et al., The Beautiful Game.

${ }^{2}$ Johnman et al., The Beautiful Game.

${ }^{3}$ Curran, Understanding the Barriers to, and Impact of Men's Engagement in Physical Activity and Health

Related Behaviours, Hunt et al., 'A Gender sensitised Weight Loss and Healthy Living Programme for

Overweight and Obese Men Delivered by Scottish Premier League Football Clubs (FFIT): A Pragmatic
}

Randomised Controlled Trial', Rutherford et al., 'Motivate'

${ }^{4} \mathrm{DH}$, Start Active Stay Active.

${ }^{5}$ WHO, Scaling up Action Against Non-Communicable Diseases: How Much Will it Cost?

${ }^{6}$ WHO, From Burden to "Best Buys": Reducing the Economic Impact of Non-Communicable Diseases in Lowand Middle Income Countries

${ }^{7}$ Zwolinsky et al., Optimizing Lifestyles for Men Regarded as "Hard-to-Reach" Through Top-Flight Football/Soccer Clubs

${ }^{8}$ WHO, From Burden to "Best Buys": Reducing the Economic Impact of Non-Communicable Diseases in Lowand Middle Income Countries.

${ }^{9}$ Clark, The Multiple Challenges of Multiple Morbidities, Lawder, Is the Scottish Population Living

Dangerously?, King, Turning Back the Clock, Nilsen, Effects of Lifestyle Intervention in Persons at Risk from Type 2 Diabetes Mellitus.

${ }^{10} \mathrm{DH}$, At Least Five a Week.

${ }^{11} \mathrm{DH}$, Start Active Stay Active.

${ }^{12}$ DH, 2011 Healthy Lives Healthy People.

${ }^{13}$ DCMS, Getting More People Playing Sport, DH, Start Active Stay Active.

${ }^{14} \mathrm{RSPH}$, Annual Conference and Awards.

${ }^{15}$ Cohen, Social Relationships and Health.

${ }^{16}$ Parnell and Richardson, Introduction.

${ }^{17} \mathrm{DH}$, Start Active Stay Active, DH Healthy Lives Healthy People.

${ }^{18}$ Hunt et al., 'A Gender sensitised Weight Loss and Healthy Living Programme for Overweight and Obese Men Delivered by Scottish Premier League Football Clubs (FFIT): A Pragmatic Randomised Controlled Trial', Pringle, and Sayers. 'It's a Goal: Basing a Community Psychiatric Nursing Service in a Local Football Stadium'.

${ }^{19}$ Parnell et al., 'Football in the Community Schemes', Pringle et al., 'Health Improvement for Men and Hardto-Engage-Men Delivered in English Premier League Football Clubs'. Rutherford et al., 'Motivate'.

${ }^{20}$ Curran, Understanding the Barriers to, and Impact of Men's Engagement in Physical Activity and Health Related Behaviours, Pringle et al., 'Health Improvement for Men and Hard-to-Engage-Men Delivered in English Premier League Football Clubs'.

${ }^{21}$ Johnman et al., The Beautiful Game.

${ }^{22}$ Parnell et al., 'Football in the Community Schemes', Pringle et al., 'Health Improvement for Men and Hardto-Engage-Men Delivered in English Premier League Football Clubs'. Rutherford et al., 'Motivate'.

${ }^{23}$ Pringle et al., The Pre-Adoption, Demographic and Health Profiles of Men Participating in a Programme of Men's Health Delivered in English Premier League Football clubs.

${ }^{24}$ Pringle et al., 'Health Improvement for Men and Hard-to-Engage-Men Delivered in English Premier League Football Clubs, Robertson et al., 'It's Fun, Fitness, Football Really.'

${ }^{25}$ Johnman et al., The Beautiful Game.

${ }^{26}$ Black, 'Can England's NHS Survive?'.

${ }^{27}$ Association of Public Service Excellence Local authority Sport and Recreation Services in England: Where next?

${ }^{28}$ Pringle et al., Assessing the Impact of Football-Based Health Improvement Programmes: Stay Onside, Avoid own Goals and Score with the Evaluation!

${ }^{29}$ Glasgow et al., REAIM. 
${ }^{30}$ Dugdill and Stratton, Evaluating Sport and Physical Activity Interventions, Pringle et al., Assessing the Impact of Football-Based Health Improvement Programmes: Stay Onside, Avoid own Goals and Score with the Evaluation!

${ }^{31}$ Curran, Understanding the Barriers to, and Impact of Men's Engagement in Physical Activity and Health Related Behaviours, Hunt et al., ' A Gender sensitised Weight Loss and Healthy Living Programme for Overweight and Obese Men Delivered by Scottish Premier League Football Clubs (FFIT): A Pragmatic Randomised Controlled Trial', Rutherford et al., 'Motivate'.

${ }^{32}$ Pringle et al., Assessing the Impact of Football-Based Health Improvement Programmes: Stay Onside, Avoid own Goals and Score with the Evaluation!

${ }^{33}$ CRI National Evaluation of LEAP, Dugdill and Stratton, Evaluating Sport and Physical Activity Interventions, National Obesity Observatory. Standard Evaluation Framework for Physical Activity Intervention, Pringle et al., Assessing the Impact of Football-Based Health Improvement Programmes: Stay Onside, Avoid own Goals and Score with the Evaluation!

${ }^{34}$ Pringle et al., Effect of a Health-Improvement Pilot Programme for Older Adults Delivered by a Professional Football Club: the Burton Albion Case Study.

${ }^{35}$ Pringle et al., Effect of a Health-Improvement Pilot Programme for Older Adults Delivered by a Professional Football Club: the Burton Albion Case Study.

${ }^{36}$ Rutherford et al., 'Motivate'.

${ }^{37}$ Ransdell et al., Developing Effective Physical Activity Programmes

${ }^{38}$ Pringle et al., Assessing the Impact of Football-Based Health Improvement Programmes: Stay Onside, Avoid own Goals and Score with the Evaluation!

${ }^{39}$ Pringle et al., Assessing the Impact of Football-Based Health Improvement Programmes: Stay Onside, Avoid own Goals and Score with the Evaluation! Pringle et al., Qualitative Perspectives on Evaluability of

Community Physical Activity Interventions'.

${ }^{40}$ Pringle et al., Assessing the Impact of Football-Based Health Improvement Programmes: Stay Onside, Avoid own Goals and Score with the Evaluation!

${ }^{41}$ The 12 Item Short form survey from the RAND Medical Outcome Study.

${ }^{42}$ Zwolinsky et al., Optimizing Lifestyles for Men Regarded as "Hard-to-Reach" Through Top-Fight Football/Soccer Clubs.

${ }^{43}$ Sport England, Learning from LEAP.

${ }^{44}$ Rutherford et al., 'Motivate'.

${ }^{45}$ Glasgow et al., REAIM.

${ }^{46}$ Pringle et al., 'Health Improvement for Men and Hard-to-Engage-Men Delivered in English Premier League Football Clubs, Robertson et al., 'It's Fun, Fitness, Football Really',

${ }^{47}$ CRI National Evaluation of LEAP

${ }^{48}$ Glasgow et al., REAIM, Pringle et al., Delivering Men's Health Interventions in English Premier League Football Clubs: Key Design Characteristics.

${ }^{49}$ Pringle et al., 'Health Improvement for Men and Hard-to-Engage-Men Delivered in English Premier League Football Clubs

${ }^{50}$ Pringle et al., Assessing the Impact of Football-Based Health Improvement Programmes: Stay Onside, Avoid own Goals and Score with the Evaluation!

${ }^{51}$ Sport England, Learning from LEAP.

${ }^{52}$ Sport England Learning from LEAP.

${ }^{53}$ Dugdill and Stratton, Evaluating Sport and Physical Activity Interventions, Sport England, Learning from LEAP. Pringle et al., Assessing the Impact of Football-Based Health Improvement Programmes: Stay Onside, Avoid own Goals and Score with the Evaluation!

${ }^{54}$ Pringle et al., Assessing the Impact of Football-Based Health Improvement Programmes: Stay Onside, Avoid own Goals and Score with the Evaluation! 
References

Association of Public Service Excellence. Local authority Sport and

Recreation Services in England: Where next?

http://www.apse.org.uk/apse/index.cfm/research/current-research-programme/local-authoritysport-and-recreation-services-in-england-where-next/local-authority-sport-and-recreationservices-in-england-where-next/ . (accessed June 6, 2014).

Black, N. 'Can England's NHS Survive?' New England Journal of Medicine 369 (2013): 30 1-3. http://www.nejm.org/doi/full/10.1056/NEJMp1305771 (accessed September 9, 2013).

Burton Albion. Head for Goal. http://burtonalbioncommunitytrust.co.uk/courses/387/ (accessed June 14, 2014).

Burton Albion Community Trust. Schools and Education. http://burtonalbioncommunitytrust.co.uk/about-schools-education/. (accessed June 6, 2014).

Carnegie Research Institute (Leeds Metropolitan University) with Matrix RCL and Ipsos MORI. National evaluation of LEAP: A Final Report on the Local Exercise Action Pilots, 2007. London: Department of Health. Available at: http://www.dh.gov.uk/en/Publicationsandstatistics/Publications/PublicationsPolicyAndGuidance/DH_073600; 2007 (accessed September 24, 2013).

Clark, N. The Multiple Challenges of Multiple Morbidities. Health Education and Behaviour 38 (2011): 219-21.

Cohen, S. Social Relationships and Health. American Psychologist 59: (2004) 676-84.

Curran, K. Understanding the Barriers to, and Impact of Men's Engagement in Physical Activity and Health Related Behaviours: An Examination of an English Premier League 
Football in the Community Men's Health Programme. PhD Thesis. Liverpool John Moores University, 2013.

Department of Culture Media and Sport. Getting More People Playing Sport. London, DCMS, 2014.

Department of Health. At Least Five a Week: Evidence on the impact of physical activity and its relationship to health. A report by the Chief Medical Officer, London: Crown, 2004.

Department of Health. Start Active, Stay Active: A Report on Physical Activity for Health from the Four Home Countries Chief Medical Officers. London: Crown, 2011. http://www.dh.gov.uk/prod_consum_dh/groups/dh_digitalassets/documents/digitalasset/dh_1 28210.pdf. (accessed 24 September 24, 2013).

Department of Health. Healthy Lives Healthy People, London, Crown, 2011.

Dugdill, L., and G. Stratton. Evaluating Sport and Physical Activity Interventions: A Guide for Practitioners. University of Salford, 2013. http://usir.salford.ac.uk/3148/1/Dugdill_and_Stratton_2007.pdf (accessed September 24, 2013).

European Commission. The State of Men's Health in Europe, Brussels, 2011.

Glasgow, R., T. Vogt, and S. Boles. 'Evaluating the Impact of Public Health Interventions: The REAIM Framework'. American Journal of Public Health 89 (1999): 1323-27.

Hunt, K., S. Wyke, C. Gray, A. Anderson, A. Brady, C. Bunn et al., 'A Gender-Sensitised Weight Loss and Healthy Living Programme for Overweight and Obese Men Delivered by Scottish Premier League Football Clubs (FFIT): A Pragmatic Randomised Controlled Trial'. The Lancet 383 (2014): 1211-24.

Johnman, C., F. Sim, and P. Mackie. 'The beautiful game.' Public Health 127 (2013): http://www.publichealthjrnl.com/article/PIIS0033350613002448/fulltext (accessed September 10, 2013). 
King, D., A. Mainous, and M. Geesey. Turning Back the Clock: Adopting a Healthy Lifestyle in Middle Age. American Journal of Medicine 120 (2007): 598-603.

Lawder, R., O. Harding, D. Stockton, C. Fischbacher, D. Brewster, J. Chalmers, A. Finlayson, and D. Conway. Is the Scottish Population Living Dangerously? Prevalence of Multiple Risk Factors: the Scottish Health Survey 2003. BMC Public Health 10 (2010): 330.

National Obesity Observatory. Standard Evaluation Framework for Physical Activity Interventions. London, National Obesity Observatory, 2012. http://www.noo.org.uk/uploads/doc/vid_16722_SEF_PA.pdf. (accessed February 20, 2014).

Nilsen, V., P Bakke, and F., Gallefoss. Effects of Lifestyle Intervention in Persons at Risk from Type 2 Diabetes Mellitus-Results from a Randomised Controlled Trial. BMC Public Health 11 (2011): 893.

Parnell, D., G. Stratton, B. Drust., and D. Richardson. 'Football in the Community Schemes: Exploring the Effectiveness of an Intervention in Promoting Positive Healthful Behaviour Change'. Soccer and Society 14 (2013): 35-51.

Parnell, D., and D. Richardson. Introduction. Soccer and Society 15 (2014): 823-8

Premier League. Creating Chances, 2011. London: Premier League. http://addison.ceros.com/premier-league/creating-chances-2011/page/1 (accessed January 25, 2013).

Pringle, A., and P. Sayers. 'It's a Goal: Basing a Community Psychiatric Nursing Service in a Local Football Stadium'. The Journal of the Royal Society for the Promotion of Health 124 (2004): 234-8.

Pringle, A., J. McKenna, E. Whatley, and N. Gilson. 'Qualitative Perspectives on Evaluability of Community Physical Activity Interventions'. From Education to Application: Sport, Exercise and Health Proceedings of the British Association of Sport \& Exercise 
Science Meeting, Wolverhampton University, Leeds, UK, British Association of Sport \& Exercise Science, September 11-13, 2006.

Pringle, A., S. Zwolinsky, A. Smith, S. Robertson, J. McKenna, and A. White. 'The Pre-Adoption Demographic and Health Profiles of Men Participating in a Programme of Men's Health Delivered in English Premier League football clubs'. Public Health 125 (2011): 411-16.

Pringle, A., S. Zwolinsky, J, McKenna, A. Smith, S. Robertson, and A. White Delivering Men's Health Interventions in English Premier League football Clubs: Key Design Characteristics. Public Health 127 (2013): 716-26.

Pringle, A., S. Zwolinsky, J. McKenna, A. Daly-Smith, S. Robertson and A. White. 'Health Improvement for Men and Hard-to-Engage-Men Delivered in English Premier League Football Clubs'. Health Education Research 29 (2014): 503-20.

Pringle, A., J. Hargreaves, L. Lozano, J. McKenna, and S. Zwolinsky. Assessing the Impact of Football-Based Health Improvement Programmes: Stay Onside, Avoid Own Goals and Score with the Evaluation! Soccer and Society 15 (2014): 970-87

Pringle, A, D. Parnell, S. Zwolinsky, J. Hargreaves, and J McKenna. Effect of a Health Improvement Pilot Programme for Older Adults Delivered by a Professional Football Club: the Burton Albion Case Study. Soccer and Society 15 (2014): 902-18.

Royal Society for Public Health. Annual Conference and Awards. Healthy settings and Developing Wellbeing in the Community. Royal Society for Public Health London, 2014. https://www.rsph.org.uk/en/courses-conferences-and-events/index.cfm/annualconference (accessed June 6, 2014).

Rutherford, Z, S. Seymour-Smith, C. Matthews, J. Wilcox, B Gough, D Parnell and A Pringle. 'Motivate': The Effect of a Football in the Community Delivered Weight Loss Programme on Over 35 year old Men and Women's Cardiovascular Risk Factors. Soccer and Society 15 (2014): 951-69. 
Sport England. Learning from LEAP. London: Sport England, 2006.

The 12 Item Short Form Survey from the RAND Medical Outcome Study. Available at: http://www.rand.org/health/surveys_tools/mos/mos_core_12item.html. (accessed June 15 2014).

World Health Organization From Burden to "Best Buys": Reducing the economic impact of non-communicable diseases in low- and middle-income countries. Geneva, Switzerland: World Health Organization. 2011.

World Health Organization Scaling Up Action Against Non-Communicable Diseases: How Much Will it Cost? Geneva, Switzerland: World Health Organization, 2011.

Zwolinsky, S., A. Pringle, A. White, A. Smith, A., S. Robertson, and J., McKenna, J Optimizing Lifestyles for Men Regarded as "Hard-to-Reach" Through Top-Flight Football/Soccer clubs. Health Education Research, 28 (2013): 405-13. 